\title{
RIGHT ENGEL ELEMENTS OF STABILITY GROUPS OF GENERAL SERIES IN VECTOR SPACES
}

\author{
B. A. F. Wehrfritz
}

\begin{abstract}
Let $V$ be an arbitrary vector space over some division ring $D, \mathbf{L}$ a general series of subspaces of $V$ covering all of $V \backslash\{0\}$ and $S$ the full stability subgroup of $\mathbf{L}$ in $\operatorname{GL}(V)$. We prove that always the set of bounded right Engel elements of $S$ is equal to the $\omega$-th term of the upper central series of $S$ and that the set of right Engel elements of $S$ is frequently equal to the hypercentre of $S$.
\end{abstract}

2010 Mathematics Subject Classification: 20F45, 20F19, $20 \mathrm{H} 25$.

Key words: Engel elements, linear groups, stability groups.

Throughout this paper we keep to the following notation. Let $V$ be a vector space over a division ring $D$ and $\mathbf{L}=\left\{\left(\Lambda_{\alpha}, V_{\alpha}\right): \alpha \in\right.$ A $\}$ a series of subspaces of $V$ running from $\{0\}$ to $V$ (see [1] for the definition and basic properties of general series). Thus in particular $\mathbf{A}$ is a linearly ordered set, the $\Lambda_{\alpha} / V_{\alpha}$ are the jumps of the series, $V \backslash\{0\}=$ $\cup_{\alpha \in \mathbf{A}} \Lambda_{\alpha} \backslash V_{\alpha}$ and $\Lambda_{\alpha} \leq V_{\beta}$ whenever $\alpha<\beta$ ( $\alpha>\beta$ just for descending series, which we usually order from the top rather than from the bottom). The completion of $\mathbf{L}$ we denote by $\mathbf{L}^{*}$. Set $S=\operatorname{Stab}(\mathbf{L})$, the full stability group of $\mathbf{L}$ in $\operatorname{GL}(V)$; that is, $S=\cap_{\alpha} C_{\mathrm{GL}(V)}\left(\Lambda_{\alpha} / V_{\alpha}\right)$. In [2] we study the set of left Engel elements of $S$. In [4] we consider the right Engel elements $S$, but only for ascending or descending series. Here we consider what we can say for general series.

Theorem 1. The set $R^{-}(S)$ of bounded right Engel elements of $S$ is equal to the $\omega$-th term $\zeta_{\omega}(S)$ of the upper central series of $S$.

In [4] we prove Theorem 1, but only in the special cases of ascending or descending series. Our proof of Theorem 1 proceeds by applying these special cases to certain ascending and descending subseries of $\mathbf{L}$. We also use Theorem 3 below in the proof of Theorem 1. Note that the left analogue of Theorem 1, namely that the set $L^{-}(S)$ of bounded left Engel elements of $S$ is equal to the Fitting subgroup $\operatorname{Fitt}(S)$ of $S$, is also valid, see [2, Theorem A]. 
The sets $R(S)$ and $L(S)$ of right and left Engel elements of $S$ are much harder to compute and we only have partial results. Suppose that either $\operatorname{dim}_{D} V$ is countable or $V$ has an $\mathbf{L}$-basis $\mathbf{B}$, meaning that $\mathbf{B} \cap L$ is a basis of $L$ for each subspace $L$ belonging to $\mathbf{L}$. Now $L(S)=\operatorname{Fitt}(S)=L^{-}(S)$, see $\left[\mathbf{2}\right.$, Theorem B], and if $\mathbf{L}$ is a descending series then $R(S)=\zeta_{\omega}(S)$, see $[\mathbf{4}, 1.2]$. However if $\mathbf{L}$ is an ascending series, then although $R(S)$ is equal to the hypercentre $\zeta(S)$ of $S$, it is frequently not equal to $\zeta_{\omega}(S)$, see $[3,1.2]$. Thus the obvious right analogue of the left Engel case is not valid, even for ascending series. However we do have the following two special cases.

Theorem 2. Suppose that either $\operatorname{dim}_{D} V$ is countable or $V$ has an $\mathbf{L}-b a$ sis. Then set $R(S)$ of right Engel elements of $S$ is equal to the hypercentre $\zeta(S)$ of $S$.

Theorem 3. Suppose either $\mathbf{L}$ has no top jump or $\mathbf{L}$ has no bottom jump (meaning that either $V=\Lambda_{\alpha}$ implies $V=V_{\alpha}$ or $\{0\}=V_{\alpha}$ implies $\left.\{0\}=\Lambda_{\alpha}\right)$. Then:

a) $R^{-}(S)=\{1\}$.

b) If either $\operatorname{dim}_{D} V$ is countable or $V$ has an $\mathbf{L}$-basis $\mathbf{B}$, then $R(S)=$ $\{1\}$.

\section{The proofs}

We keep our notation above, in particular we have our series $\mathbf{L}$ and $\mathbf{L}^{*}$ and stability group $S$. We always assume that $V$ is a left vector space over $D$. Clearly there are analogous results for right vector spaces. Also clearly we may remove all trivial jumps $\Lambda_{\alpha}=V_{\alpha}$ from $\mathbf{L}$ without affecting the conclusions of the theorems. Thus below assume that $\Lambda_{\alpha}>V_{\alpha}$ for all $\alpha$ in $\mathbf{A}$. This makes various statements simpler. For example, the hypothesis of Theorem 3 is now that $\mathbf{A}$ has either no maximal member or no minimal member. Let Fitt $(L)$ denote the set of elements of $S$ that stabilize some finite subseries of $\mathbf{L}^{*}$ running from $\{0\}$ to $V$; $\operatorname{Fitt}(L)$ is a normal subgroup of $S$ contained in $\operatorname{Fitt}(S)$.

Lemma 1. Let $x \in R(S) \cap \operatorname{Fitt}(\mathbf{L})$ and set $X=\cup\left\{\Lambda_{\alpha}: \Lambda_{\alpha}(x-1)=\right.$ $\{0\}\} ; X$ is an element of $\mathbf{L}^{*}$. Then $\mathbf{C}=\left\{\alpha \in \mathbf{A}: X \leq V_{\alpha}\right\}$ is inversely well-ordered (so $\left\{\left(\Lambda_{\alpha}, V_{\alpha}\right): X \leq V_{\alpha}\right\}$ is a descending series).

Proof: Clearly we may assume that $x \neq 1$. Now $x$ stabilizes a finite subseries $\{0\}=X_{0}<X_{1}<\cdots<X_{r}=V$ of $\mathbf{L}^{*}$, where clearly $r \geq 2$ and we may assume that $X_{1}=X$. If $\mathbf{C}$ is not inversely well-ordered there exist a $j \geq 1$ and an infinite subsequence $\alpha(1)<\alpha(2)<\cdots<\alpha(i)<\cdots$ 
of $\mathbf{C}$ with $X_{j} \leq V_{\alpha(1)}$ and $Y=\cup_{i} \Lambda_{\alpha(i)} \leq X_{j+1}$. Let $\mathbf{M}$ denote the ascending subseries

$$
\begin{aligned}
\{0\}<X_{1}<\cdots<X_{j} \leq V_{\alpha(1)}<\Lambda_{\alpha(1)} & \leq \cdots \leq V_{\alpha(i)} \\
& <\Lambda_{\alpha(i)} \leq V_{\alpha(j+1)}<\cdots \leq Y
\end{aligned}
$$

of $\mathbf{L}^{*}$. Clearly $\left.x\right|_{Y} \in \operatorname{Stab}(\mathbf{M})=T$ say and $T \leq \operatorname{GL}(Y)$. Choose a subspace $W$ of $V$ with $V=W \oplus Y$ and extend the action of $T$ on $Y$ to one on $V$ by making $T$ centralize $W$. Then $T \leq S$. Also if $t \in T$, then $\left.\left[\left.x\right|_{Y},{ }_{k} t\right]\right|_{Y}=\left.\left[x,{ }_{k} t\right]\right|_{Y}$ for all $k \geq 1$ and so $\left.x\right|_{Y} \in R(T)$. But then $\left.x\right|_{Y}=1$ by 1.1c) of [4]. This contradicts the choice of $X_{1}$ and completes the proof of Lemma 1.

Lemma 2. Let $x \in R(S)$ and define $X$ and $\mathbf{C}$ as in Lemma 1.

a) If $x \in R^{-}(S)$ then $\mathbf{C}$ is inversely well-ordered.

b) If either $\operatorname{dim}_{D} V$ is countable or $V$ has an $\mathbf{L}$-basis, then $\mathbf{C}$ is inversely well-ordered.

Proof: a) $R^{-}(S) \subseteq L^{-}(S)^{-1} \subseteq \operatorname{Fitt}(\mathbf{L})$ by $[\mathbf{1}, 7.11]$ and [2, Theorem A]. Thus Lemma 1 applies.

b) Here $R(S) \subseteq L(S)^{-1} \subseteq \operatorname{Fitt}(\mathbf{L})$ by $[\mathbf{1}, 7.11]$ and $[\mathbf{2}$, Theorem B] and again Lemma 1 applies.

Lemma 3. Let $x \in R^{-}(S) \cap$ Fitt $(\mathbf{L})$ and set $X=\cap\left\{V_{\alpha}: V(x-1) \leq V_{\alpha}\right\}$; $X$ is an element of $\mathbf{L}^{*}$. Then $\mathbf{C}=\left\{\alpha \in \mathbf{A}: \Lambda_{\alpha} \leq X\right\}$ is well-ordered (so $\left\{\left(\Lambda_{\alpha}, V_{\alpha}\right): \Lambda_{\alpha} \leq X\right\}$ is an ascending series).

Proof: Again assume that $x \neq 1$, so $x$ stabilizes a finite subseries $\{0\}=$ $X_{0}<X_{1}<\cdots<X_{r}=V$ of $\mathbf{L}^{*}$, where $r \geq 2$ and $X_{r-1}=X$. If $\mathbf{C}$ is not well-ordered there exist a $j<r$ and an infinite subsequence $\alpha(1)>$ $\alpha(2)>\cdots .>\alpha(i)>\cdots$ of $\mathbf{C}$ with $X_{j} \geq \Lambda_{\alpha(1)}$ and $Y=\cap_{i} V_{\alpha(i)} \geq X_{j+1}$. Let $\mathbf{M}$ denote the descending subseries of $V / Y$ consisting of the $X_{k} / Y$ for $j \leq k \leq r$ and the $\Lambda_{\alpha(i)} / Y$ and the $V_{\alpha(i)} / Y$ for $i \geq 1$. (This is a descending subseries of length $\omega$ of $\mathbf{L}^{*}$ taken modulo the element $Y$ of $\mathbf{L}^{*}$.)

Clearly $\left.x\right|_{V / Y} \in T=\operatorname{Stab}(\mathbf{M})$. Pick a subspace $W$ of $V$ with $V=$ $W \oplus Y$ and let $T$ act on $W$ via its given action on $V / Y$ and the natural isomorphism of $V / Y$ onto $W$. Then extend this action of $T$ on $W$ to one on $V$ by making $T$ centralize $Y$. Clearly $T \leq S$ and $\left.\left[\left.x\right|_{V / Y},{ }_{k} t\right]\right|_{V / Y}=$ $\left.\left[x,{ }_{k} t\right]\right|_{V / Y}$ for all $k \geq 1$ and all $t \in T$. Hence $\left.x\right|_{V / Y} \in R^{-}(T)$. But then $\left.x\right|_{V / Y} \in \zeta(T)$ by $\left.[4,1.2 \mathrm{a})\right]$ and $\zeta(T)=\langle 1\rangle$ by $[\mathbf{3}, 1.1]$. Lemma 3 follows. 
Lemma 4. If $x \in R^{-}(S)$ and if $\mathbf{C}$ is as in Lemma 3, then $\mathbf{C}$ is well-ordered.

Proof: Now $R^{-}(S) \subseteq \operatorname{Fitt}(\mathbf{L})$ by $[\mathbf{1}, 7.11]$ and $[\mathbf{2}$, Theorem A]. Thus Lemma 3 applies.

Lemma 5. Suppose either $\operatorname{dim}_{D} V$ is countable or $V$ has an $\mathbf{L}$-basis. If $x \in R(S)$ and if $X=\cap\left\{V_{\alpha}: V(x-1) \leq V_{\alpha}\right\}$, then $\mathbf{C}=\left\{\alpha \in \mathbf{A}: \Lambda_{\alpha} \leq\right.$ $X\}$ is well-ordered.

Proof: As before $R(S) \subseteq L(S)^{-1} \subseteq \operatorname{Fitt}(L)$ by [1, 7.11] and [2, Theorem B]. Now repeat the proof of Lemma 3. Here we only obtain that $\left.x\right|_{V / Y} \in R(T)$. But here $R(T)=\{1\}$ by $\left.[\mathbf{4}, 1.2 \mathrm{c})\right]$. Lemma 5 now follows.

Lemma 6. Suppose that A either has no maximal member or has no minimal member. Then $R^{-}(S)=\{1\}$. If also either $\operatorname{dim}_{D} V$ is countable or $V$ has an $\mathbf{L}$-basis, then $R(S)=\{1\}$.

This lemma completes the proof of Theorem 3.

Proof: If $\mathbf{A}$ has no maximal member, then in Lemma 2 always $\mathbf{C}$ is empty. Thus in Lemma 2, case a) we have $R^{-}(S)=\{1\}$ and in Lemma 2, case b) we have $R(S)=\{1\}$.

Now assume $\mathbf{A}$ has no minimal member. Then $\mathbf{C}$ is always empty in Lemmas 4 and 5 . Thus in Lemma 4 we have $R^{-}(S)=\{1\}$ and in Lemma 5 we have $R(S)=\{1\}$. Lemma 6 follows.

Further notation. The series $\mathbf{L}^{*}$ has a maximal ascending segment starting at $\{0\}$; specifically there is an ordinal number $\lambda=\mu+n$, where $n \geq 0$ is an integer, $\mu$ is zero or a limit ordinal, and

$$
\{0\}=\Lambda_{0}<\Lambda_{1}<\cdots<\Lambda_{\gamma}<\cdots<\Lambda_{\lambda}
$$

is a subseries of $\mathbf{L}^{*}$ with each $\Lambda_{\gamma+1} / \Lambda_{\gamma}$ a jump of $\mathbf{L}$ and $\Lambda_{\lambda} \neq V_{\alpha}$ for all $\alpha \in \mathbf{A}$.

In the same way $\mathbf{L}^{*}$ has a maximal descending segment

$$
V=V_{0}>V_{1}>\cdots>V_{\gamma}>\cdots>V_{\lambda^{\prime}},
$$

where $\lambda^{\prime}=\mu^{\prime}+n^{\prime}$ is an ordinal number, $n^{\prime} \geq 0$ is an integer, $\mu^{\prime}$ is zero or a limit ordinal, each $V_{\gamma} / V_{\gamma+1}$ is a jump of $\mathbf{L}$ and $V_{\lambda^{\prime}} \neq \Lambda_{\alpha}$ for all $\alpha \in \mathbf{A}$.

Clearly $V_{\mu^{\prime}} \geq \Lambda_{\lambda}$ and $V_{\lambda^{\prime}} \geq \Lambda_{\mu}$. Thus we have two possibilities. Firstly we could have that $V_{\lambda^{\prime}}>\Lambda_{\lambda}$. Secondly if this is not the case then $V_{\mu^{\prime}}=\Lambda_{\lambda}$ and $V_{\lambda^{\prime}}=\Lambda_{\mu}$. 
Proof of Theorem 1: We have to prove that $R^{-}(S) \subseteq \zeta_{\omega}(S)$. By [4, 1.1 and 1.2] we may assume that $\mathbf{L}$ is neither an ascending series nor a descending series. Set $R=R^{-}(S)$. Then $R$ is a subgroup of $S$ by [4, Theorem 1.3] and $R$ centralizes $V / \Lambda_{\lambda}$ and $V_{\lambda^{\prime}}$ by Theorem 3 (and [2, 1.5]). Thus if $x \in R$, then $x \phi: v \mapsto v(x-1)$ is a linear homomorphism of $V$ into $\Lambda_{\lambda}$ with $V_{\lambda^{\prime}} \leq \operatorname{ker}(x \phi)$. Thus $\phi$ is effectively an embedding of $R$ into $\operatorname{Hom}_{D}\left(V / V_{\lambda^{\prime}}, \Lambda_{\lambda}\right)$. We are not claiming at this stage that $\phi$ is a homomorphism of $R$; that is, possibly $(x y) \phi \neq x \phi+y \phi$, for some $x$ and $y$ in $R$.

Let $\mathbf{M}$ denote the descending subseries $V=V_{0}>V_{1}>\cdots>V_{\gamma}>$ $\cdots>V_{\lambda^{\prime}}>\{0\}$ of $\mathbf{L}^{*}$. Then $R \leq T=\operatorname{Stab}(\mathbf{M}) \leq S$, so $R \leq R^{-}(T)$. Then $R \leq \zeta_{\omega}(T)$ by $[\mathbf{4}, 1.2]$ and hence by $[\mathbf{3}, 4.1]$ (and its proof) $R \phi$ is contained in the subset of $\operatorname{End}_{D} V$ of all $\theta$ such that $V \theta \leq V_{\mu^{\prime}}$ and there exists $i<\omega$ with $V_{i} \theta=\{0\}$. (Possibly $V_{\mu^{\prime}}=V$, in which case $\mu^{\prime}=0$ and we can set $i=\lambda^{\prime}$.)

Let $\mathbf{N}$ denote the ascending subseries $\{0\}=\Lambda_{0}<\Lambda_{1}<\cdots<\Lambda_{\gamma}<$ $\cdots<\Lambda_{\lambda}<V$ of $\mathbf{L}^{*}$. Then $R \leq R^{-}(U)$ for $U=\operatorname{Stab}(\mathbf{N})$ and so $R \leq \zeta_{\omega}(U)$ by $[\mathbf{4}, 1.1]$. Consequently if $\theta \in R \phi$, then for some $j<\omega$ we have $V \theta \leq \Lambda_{j}$ (and $\Lambda_{\mu} \theta=\{0\}$, which here we already know), see the last line of the proof of 3.5 of [4], where $\zeta_{\omega}(U)$ is identified, in the notation of [4], with $T_{\omega_{-}}$. Again, if $\Lambda_{\mu}=\{0\}$, then $\mu=0$ and we can choose $j=\lambda$.

Set $K_{i j}=\left\{\theta \in \operatorname{End}_{D} V: V_{i} \theta=\{0\}\right.$ and $\left.V \theta \leq \Lambda_{j}\right\}$, where $0 \leq i, j<$ $\omega, i \leq \lambda^{\prime}$, and $j \leq \lambda$. If $\theta \in K_{i j}, v \in V$, and $g \in S$, then

$$
v[\theta, g]=v g^{-1} \theta g-v \theta=v\left(g^{-1}-1\right) \theta g+v \theta(g-1) .
$$

If also $i \geq 1$, then $V_{i-1}\left(g^{-1}-1\right) \theta g \leq V_{i} \theta g=\{0\}$ and $V\left(g^{-1}-1\right) \theta g \leq$ $V \theta g \leq \Lambda_{j}$. Further if $j \geq 1$, then $V \theta(g-1) \leq \Lambda_{j}(g-1) \leq \Lambda_{j-1}$ and $V_{i} \theta(g-1)=\{0\}$. Consequently $\left[K_{i j}, S\right] \leq K_{i-1, j}+K_{i, j-1}$ whenever $i, j \geq 1$. Also $K_{i j}=\{0\}$ if either $i=0$ or $j=0$. Set $L_{r}=\sum_{i+j \leq r} K_{i j}$. Then

$$
\{0\}=L_{0}=L_{1} \leq L_{2} \leq \cdots \leq L_{r} \leq \cdots
$$

with $\left[L_{r+1}, S\right] \leq L_{r}$ for all $r \geq 1$. Also $R \phi \subseteq L=\cup_{r} L_{r}$.

Suppose $\mu^{\prime}$ is infinite. Then all $V_{i} \geq V_{\mu^{\prime}}$ and $\left[V_{\mu^{\prime}}, R\right]=\{0\}$; also $[V, R] \leq \Lambda_{\lambda} \leq V_{\mu^{\prime}}$. Thus in this case $\phi$ is an $S$-monomorphism of $R$ and hence $R \leq \zeta_{\omega}(S)$. Suppose $\mu$ is infinite. Then all $\Lambda_{j} \leq \Lambda_{\mu}$ and $[V, R] \leq \Lambda_{\mu}$; also $\left[\Lambda_{\mu}, R\right] \leq\left[V_{\lambda^{\prime}}, R\right]=\{0\}$. Thus here too $\phi$ is an $S$-monomorphism of $R$ and again $R \leq \zeta_{\omega}(S)$. Finally suppose $\mu=$ $0=\mu^{\prime}$. Then $\Lambda_{\lambda}<V_{\lambda^{\prime}}$ (recall $\mathbf{L}$ here is not ascending, or for that matter descending). But then $[V, R] \leq \Lambda_{\lambda},\left[V_{\lambda^{\prime}}, R\right]=\{0\}, \phi$ is an $S$ monomorphism and $R \leq \zeta_{\omega}(S)$ (actually in this case $R \leq \zeta_{\lambda+\lambda^{\prime}}(S)$ ). 
Proof of Theorem 2: Here $\operatorname{dim}_{D} V$ is countable or $V$ has an L-basis. As far as we can, we follow the strategy of the proof of Theorem 1. Let $R=R(S)$. We need only prove that $R \subseteq \zeta(S)$. Again we may assume that $\mathbf{L}$ is neither ascending nor descending by $[\mathbf{4}, 1.1$ and 1.2]. Also $R$ is a normal subgroup of $S$ by $[4,1.3]$ and $R$ centralizes $V / \Lambda_{\lambda}$ and $V_{\lambda^{\prime}}$ by Theorem 3. If $x \in R$ and $x \phi: v \mapsto v(x-1)$, then $\phi$ embeds $R$ into $\operatorname{Hom}_{D}\left(V / V_{\lambda^{\prime}}, \Lambda_{\lambda}\right)$, which we regard as a subset of $\operatorname{End}_{D}(V)$ in the usual way.

With $\mathbf{M}$ and $T=\operatorname{Stab}(\mathbf{M})$ as in the proof of Theorem 1, we have $R \leq T \leq S$ and $R \leq R(T)=\zeta_{\omega}(T)$, the latter by $[\mathbf{4}, 1.2]$. Hence $[\mathbf{3}, 4.2]$ yields that for each $x$ in $R$ there exists $i<\omega$ with $V_{i}(x-1)=\{0\}$. Again if $\mu^{\prime}=0$ then $\lambda^{\prime}$ is finite and we can choose $i=\lambda^{\prime}$ for all such $x$. To avoid two formally different cases, if $\mu^{\prime}=0$ set $V_{i}=V_{\lambda^{\prime}}$ for $\lambda^{\prime}<i \leq \omega$. Thus in both cases we have $R \phi \subseteq \operatorname{Hom}_{D}\left(V / V_{\omega}, \Lambda_{\lambda}\right)$; in fact we have

$$
R \phi \subseteq \bigcup_{i<\omega} \operatorname{Hom}_{D}\left(V / V_{i}, \Lambda_{\lambda}\right)
$$

Let $\mathbf{N}$ be as in the proof of Theorem 1 and again set $U=\operatorname{Stab}(\mathbf{N})$. Then $R \leq R(U)=\zeta(U)$ by $[\mathbf{4}, 1.1]$. From now on we can no longer proceed as in the proof of Theorem 1 since $\zeta(U)$ has a more complicated structure than $\zeta_{\omega}(U)$. Set $P=R \cap C_{U}\left(V / \Lambda_{\mu}\right)$. Then $P \leq C_{U}\left(V_{\lambda^{\prime}}\right) \leq$ $C_{U}\left(\Lambda_{\mu}\right)$ and $V_{\omega} \geq \Lambda_{\mu}$. Hence $\phi: P \rightarrow \operatorname{Hom}_{D}\left(V / V_{\omega}, \Lambda_{\mu}\right)$ is a group embedding.

Let $H_{\gamma}=\operatorname{Hom}_{D}\left(V / V_{\omega}, \Lambda_{\gamma}\right)$. Then $\left\{P \phi \cap H_{\gamma}\right\}_{\gamma \leq \mu}$ is an ascending $U$-hypercentral series of $P \phi$ by [3, 3.2 and 3.3]. Also $\left(P \phi \cap H_{\gamma+1}\right) /(P \phi \cap$ $\left.H_{\gamma}\right)$ embeds into $\operatorname{Hom}_{D}\left(V / V_{\omega}, \Lambda_{\gamma+1} / \Lambda_{\gamma}\right)$. Let $K_{i}=\operatorname{Hom}_{D}\left(V / V_{i}, \Lambda_{1}\right)$. Now $P \phi \cap H_{1} \leq \cup_{i<\omega} K_{i}$ since for each $x \in R$ there exists $i<\omega$ with $V(x-1) \leq V_{i}$. Also $K_{i+1} / K_{i} \cong \operatorname{Hom}_{D}\left(V_{i} / V_{i+1}, \Lambda_{1}\right)$ and the latter is centralized by $S$. Consequently $P \phi \cap H_{1}$ is $S$-hypercentral. In view of $[2,1.5]$ we may apply this to $V / \Lambda_{\gamma}$ for each $\gamma<\mu$ and thus each $\left(P \phi \cap H_{\gamma+1}\right) /\left(P \phi \cap H_{\gamma}\right)$ is $S$-hypercentral. Therefore $P \leq \zeta(S)$.

We now need to consider $R / P$. This acts faithfully on $V / \Lambda_{\mu}$. In view of $[2,1.5]$ we may simplify our notation by assuming that $\Lambda_{\mu}=\{0\}$; that is, that $\mu=0$. If $V_{\lambda^{\prime}}=\Lambda_{\mu}$ then $\mathbf{L}$ is a descending series; in which case we already know that $R \leq \zeta(S)$. If $V_{\lambda^{\prime}} \neq \Lambda_{\mu}$, then we have $V \geq V_{\omega} \geq V_{\mu^{\prime}} \geq V_{\lambda^{\prime}}>\Lambda_{\lambda} \geq \Lambda_{\mu}=\{0\}$. Also $[V, R] \leq \Lambda_{\lambda}$ and $\left[V_{\omega}, R\right]=$ $\{0\}$. Consequently $\phi: R \rightarrow \operatorname{Hom}_{D}\left(V / V_{\omega}, \Lambda_{\lambda}\right)$ is a group embedding. But in fact $\phi$ embeds $R$ into $\cup_{i<\omega} \operatorname{Hom}_{D}\left(V / V_{i}, \Lambda_{\lambda}\right)$ and the latter is $S$-hypercentral; it has an ascending series with factors isomorphic to the $S$-trivial modules $\operatorname{Hom}_{D}\left(V_{i} / V_{i+1}, \Lambda_{j+1} / \Lambda_{j}\right)$. Consequently $R \leq \zeta(S)$ and the proof of Theorem 2 is complete. 


\section{References}

[1] D. J. S. Robinson, "Finiteness Conditions and Generalized Soluble Groups", Parts 1 and 2, Ergebnisse der Mathematik und ihrer Grenzgebiete 62 and 63, Springer-Verlag, New York-Berlin, 1972.

[2] B. A. F. Wehrfritz, Stability groups of series in vector spaces, $J$. Algebra 445 (2016), 352-364. DOI: 10.1016/j. jalgebra.2015.09.006.

[3] B. A. F. Wehrfritz, The central heights of stability groups of series in vector spaces, Czechoslovak Math. J. 66(141) (2016), no. 1, 213-222. DOI: $10.1007 / \mathrm{s} 10587-016-0251-4$.

[4] B. A. F. Wehrfritz, Right Engel elements of stability groups of series in vector spaces, J. Pure Appl. Algebra 220(7) (2016), 2701-2710. DOI: 10.1016/j.jpaa.2015.12.006.

School of Mathematical Sciences

Queen Mary University of London

London E1 4NS

England

E-mail address: b.a.f.wehrfritz@qmul.ac.uk

Rebut el 27 d'agost de 2015. 